\title{
The role of intestinal stoma in patients with spinal cord injury
}

\author{
SR Kelly ${ }^{1}$, M Shashidharan ${ }^{1}$, B Borwell ${ }^{1}$, AM Tromans ${ }^{2}$, D Finnis ${ }^{1}$ and DJ Grundy ${ }^{2}$ \\ ${ }^{1}$ Department of General Surgery, ${ }^{2}$ The Duke of Cornwall Spinal Treatment Centre, Salisbury, UK
}

In the long term following spinal cord injury, bowel dysfunction causes major physical and psychological problems. A retrospective review of intestinal stomas performed in patients with spinal cord injury over a 10 year period was performed to investigate their role in alleviating these problems. Twelve patients underwent left iliac fossa end colostomy and two patients right iliac fossa end ileostomy. The mean age of patients at operation was 54.8 years $(20-65)$, and the mean time from injury to stoma formation was 15 years $(2-37)$. The mean period of unsatisfactory bowel management before stoma formation was 5.4 years $(1.5-25)$. Following colostomy the mean time spent on bowel care per week fell from $8.8 \mathrm{~h}(0.6-12.2)$ to $1.4 \mathrm{~h}$ $(0.3-3.5)$ and independence in bowel care rose from 50 to $92 \%$. All patients stated that their bowel care was easier and $83 \%$ said their independence had increased. Ninety-two per cent wished colostomy had been offered earlier and no patient undergoing colostomy wanted it reversed. The most common complication following colostomy was exclusion colitis. The mean time of follow-up post colostomy formation was 38 months (7-130). Formation of an intestinal stoma is a safe, effective and well accepted treatment for selected patients with intractable problems of bowel management following spinal cord injury.

Keywords: intestinal stoma; quality of life; spinal cord injury

\section{Introduction}

Following spinal cord injury, it is not only the obvious motor and sensory loss, but the adverse effects on bladder, bowel and sexual function that concern the patient. Recent studies $^{1-5}$ have agreed that bowel dysfunction is a major physical and psychological problem in these patients.

The standard bowel care regimen varies according to the level of the lesion. In upper motor neurone lesions reflex evacuation may be produced using digital stimulation or the insertion of glycerine suppositories followed by manual or spontaneous reflex evacuation. In paraplegics this can usually be done independently over the toilet. Most tetraplegics need assistance and, as their balance is not always good enough for this to be performed over the toilet, it is often performed in bed. In patients with lower motor neurone lesions suppositories are ineffective and manual evacuation is normally carried out.

As life expectancy in spinal cord injured patients improves, severe bowel-associated problems including constipation, faecal incontinence and abdominal distension are likely to be increasingly seen and elective surgical treatments such as colostomy ${ }^{6}$, anterior sacral root stimulation ${ }^{7}$ and antegrade colonic lavage ${ }^{8}$ have been proposed.

Correspondence: DJ Grundy, The Duke of Cornwall Spinal Treatment Centre, Salisbury District Hospital, Salisbury SP2 8BJ, UK
There is no agreement as to the timing of stoma formation for chronic gastrointestinal problems following spinal cord injury and only two studies give the results of colostomy for these indications. ${ }^{5,6}$

We reviewed our experience of intestinal stomas performed on spinal cord injured patients at The Spinal Treatment Centre at Salisbury District Hospital over a 10 year period (1986-1996), to ascertain the problems patients experienced with bowel management prior to intestinal stoma formation, and its effects on quality of life. We also studied the complications associated with the procedures.

\section{Patients and methods}

Fourteen patients with intractable bowel problems, 12 males and two females, underwent colostomy or ileostomy between 1993 and 1996 except one patient who was operated on in 1986. All patients were seen preoperatively by a stoma nurse and the position of the stoma was marked with the patient sitting in his/her wheelchair. Each patient was admitted three days preoperatively and given 4 litres of polyethylene glycol solution (Klean-Prep) and clear fluids as bowel preparation.

Twelve patients were treated with left iliac fossa end colostomy and two with right iliac fossa end ileostomy. One patient undergoing ileostomy had it as his first operation, the other had a previous colostomy for constipation and underwent ileostomy and total colectomy for continued constipation and 
perianal pain. Only one other patient in the group underwent bowel resection, having an abdominoperineal resection for primary treatment of anal cancer. In the remainder the distal bowel was closed.

Of the 12 patients treated with colostomy three had cervical lesions, eight thoracic lesions and one a lumbar lesion. Both patients treated with ileostomy had thoracic lesions (Table 1). The cause of injury of those treated with colostomy was traumatic in ten patients, due to transverse myelitis in one and following arachnoiditis and surgery for spinal stenosis in one. Both patients treated with ileostomy had traumatic causes of their spinal cord injury.

The mean age of patients at injury was 34.8 years (34.5 for colostomy and 43.0 for ileostomy). The mean age at operation was 54.8 years ( 54.5 for colostomy and 57 for ileostomy) (Table 1). The mean time from injury to stoma formation was 15 years (15.2 years for colostomy and 14.0 years for ileostomy) (Table 1).

All patients were sent a stoma questionnaire. We used Stone's classification describing the overall effect stoma formation had on their quality of life: $1=$ much worse, $2=$ worse; $3=$ unchanged; $4=$ better; $5=$ much better.
Any complications of the procedures performed and confirmation of questionnaire data were obtained by case note review.

\section{Results}

The indications for stoma formation are given in Table 2 .

All but one of the operations took place from 1993-1996 and this was due to a change in practice, in offering an intestinal stoma earlier to patients having major problems with bowel care.

The mean period of satisfactory bowel management after spinal cord injury was 9.5 years (9.6 years for those having a colostomy, 9.0 years for those having an ileostomy). The mean period of unsatisfactory bowel management before stoma formation was 5.4 years (5.7 years for the colostomy group, 3.5 years for the ileostomy group) (Table 1).

Of those patients treated with colostomy, the mean time spent on bowel care per week before stoma formation was $8.8 \mathrm{~h}(0.6-12.2)$ compared with $1.4 \mathrm{~h}$ $(0.3-3.5)$ after stoma formation (Figure 1) $50 \%$ of these patients were independent in bowel care before

Table 1 Patient details

\begin{tabular}{|c|c|c|c|c|c|c|}
\hline $\begin{array}{l}\text { Patient } \\
\text { No. }\end{array}$ & $\begin{array}{l}\text { Neurological } \\
\text { level }\end{array}$ & Sex & $\begin{array}{c}\text { Age at stoma } \\
\text { formation (years) }\end{array}$ & $\begin{array}{l}\text { Time from injury } \\
\text { to stoma (years) }\end{array}$ & $\begin{array}{l}\text { Period of satisfactory bowel } \\
\text { management (years) }\end{array}$ & $\begin{array}{l}\text { Period of unsatisfactory } \\
\text { bowel management (years) }\end{array}$ \\
\hline \multicolumn{7}{|c|}{ Colostomy } \\
\hline 1 & T6 & M & 65 & 9 & 4.5 & 4.5 \\
\hline 2 & L2 & $\mathrm{F}$ & 64 & 2 & 0 & 2 \\
\hline 3 & C6 & $\mathrm{M}$ & 20 & 3 & 1.5 & 1.5 \\
\hline 4 & $\mathrm{~T} 4$ & $\mathrm{~F}$ & 58 & 37 & 35 & 2 \\
\hline 5 & $\mathrm{~T} 4$ & $\mathrm{M}$ & 33 & 4 & 2 & 2.5 \\
\hline 6 & C6 & $\mathrm{M}$ & 60 & 36 & 34 & 2 \\
\hline 7 & $\mathrm{C} 4$ & $\mathrm{M}$ & 63 & 25 & 22 & 3 \\
\hline 8 & T5 & $\mathrm{M}$ & 61 & 7 & 0 & 7 \\
\hline 9 & $\mathrm{~T} 11$ & $\mathrm{M}$ & 49 & 29 & 4 & 25 \\
\hline 10 & $\mathrm{~T} 4$ & $\mathrm{M}$ & 36 & 11 & 1.75 & 9.25 \\
\hline 11 & $\mathrm{~T} 10$ & $\mathrm{M}$ & 60 & 9 & 3 & 7 \\
\hline 12 & $\mathrm{~T} 7$ & $\mathrm{M}$ & 28 & 10 & 7 & 3 \\
\hline \multicolumn{7}{|c|}{ Ileostomy } \\
\hline 13 & $\mathrm{~T} 4$ & $\mathrm{M}$ & 56 & 9 & 4 & 2 \\
\hline 14 & $\mathrm{~T} 4$ & $\mathrm{M}$ & 58 & 19 & 14 & 5 \\
\hline
\end{tabular}

Summary: 3 Cervical, 10 Thoracic, 1 Lumbar lesion. 12 males and 2 females. Average age 54.8 years (Colostomy 54.5, Ileostomy 57.0). Mean period of time from injury to stoma formation: 15 years; mean period of satisfactory bowel management before stoma: 9.5 years; Mean period of unsatisfactory bowel management before stoma 5.4 years

Table 2 Indications for stoma formation

\begin{tabular}{lll}
\hline Colostomy & Constipation & $8(2$ with chronic sigmoid volvulus $)$ \\
& Abdominal pain & 2 \\
& Incontinence & 1 \\
Sleostomy & Squamous cell carcinoma of anus & 1 \\
& Constipation & 1 \\
\hline
\end{tabular}


the operation, but this figure rose to $92 \%$ following colostomy formation. The only patient still dependent on a carer was a $\mathrm{C} 4$ tetraplegic.

All patients stated that their bowel care was easier after stoma formation and $83 \%$ said their independence had increased. Ten patients claimed that the colostomy had a very beneficial effect on their quality of life (Table 3). Overall colostomy formation was well accepted with $92 \%$ wishing it had been offered earlier and no patient wanting the operation to be reversed. The mean follow-up post colostomy formation was $38.0(7-130)$ months.

In the two patients treated with ileostomy, the mean time spent on bowel care per week before ileostomy was $17.5 \mathrm{~h}$ and this was unchanged after ileostomy formation. Both were independent in bowel care before the operation. In one patient it was his first operation for constipation following spinal cord injury. He had suffered severe constipation for 5 years before his ileostomy, spending $4 \mathrm{~h}$ a day on bowel care. After the decision to peform an intestinal stoma was made, he requested an ileostomy as a close

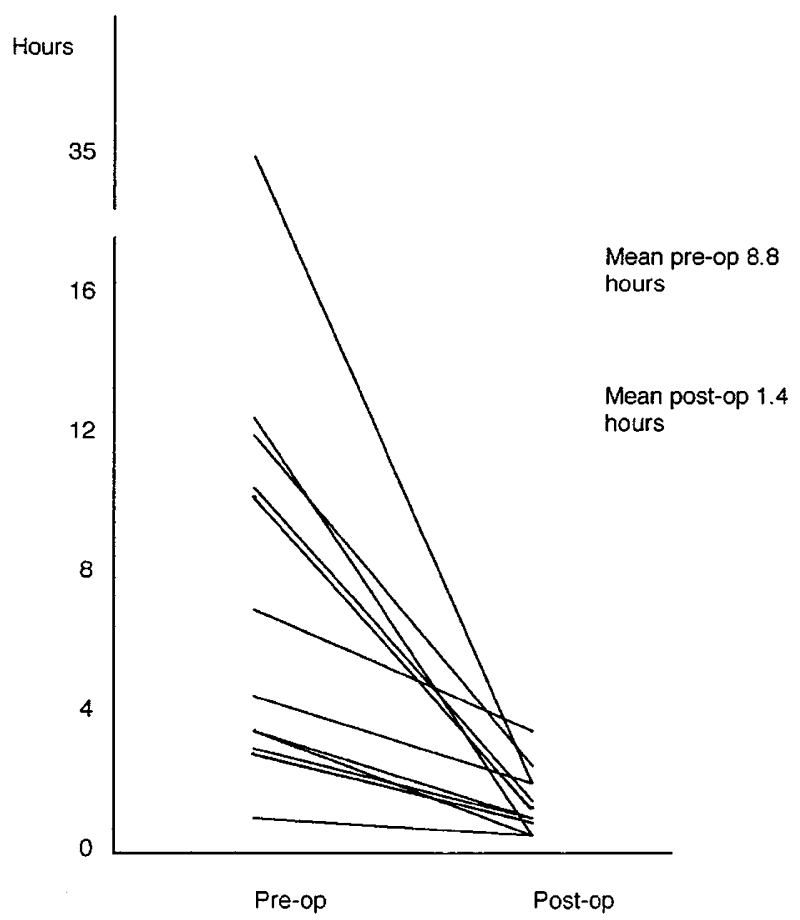

Figure 1 Time spent on bowel care pre and post colostomy

Table 3 Effect of stoma on quality of life

\begin{tabular}{lcc}
\hline & Colostomy & Ileostomy \\
\hline Much worse & 0 & 0 \\
Worse & 1 & 1 \\
Unchanged & 0 & 0 \\
Better & 1 & 0 \\
Much better & 10 & 1 \\
\hline
\end{tabular}

friend had been successfully managed by one. It decreased the time he spent on bowel care each week from $28 \mathrm{~h}$ to $14 \mathrm{~h}$, making it much easier and making him independent in bowel care for the first time since his injury.

The other patient had a colostomy performed for constipation in South Africa in 1990, 4 years after his spinal cord injury. This decreased the time spent on bowel care from $21 \mathrm{~h}$ a week to $7 \mathrm{~h}$ a week, although he felt his bowel care was easier before his colostomy. However he developed severe rectal pain and problems with constipation with his colostomy. He underwent a subtotal colectomy and ileostomy. Following this his time spent on bowel care per week increased again to $21 \mathrm{~h}$ and he is troubled by large volumes of effluent from his ileostomy. He feels his bowel care was easier before the ileostomy and his quality of life, which was unchanged following the colostomy is now worse. He is the only patient in the study who wished the operation to be reversed. The mean follow-up post ileostomy was $8.5(8-9)$ months.

There was one death in the colostomy group, a man who had squamous cell carcinoma of the anus and died from his disease. Two patients developed exclusion colitis, one of whom underwent excision of the rectal stump but developed a faecal fistula to the abdominal wound, which settled after a defunctioning loop ileostomy. One patient developed obstruction at the site of the extraperitoneal tunnel immediately proximal to the colostomy in the initial post operative period. This was resolved at laparotomy by dividing the extraperitoneal tunnel. One patient had continuing constipation.

In the ileostomy group, the patient who had a preexisting colostomy and underwent ileostomy and subtotal colectomy developed a pelvic abscess which required a perineal proctectomy, from which he made a good recovery.

\section{Discussion}

These results show that formation of an intestinal stoma, principally left iliac fossa end colostomy, is a successful treatment option for patients with intractable problems of bowel management in the longer term following spinal cord injury.

The period of satisfactory bowel management following spinal cord injury from our study is just below 10 years. This corresponds to other studies on bowel function in spinal cord injury patients ${ }^{1,2}$ which showed that, although bowel function was abnormal, no patients had undergone surgery in these studies as the follow up periods were well below 10 years.

Colostomy markedly decreased the time spent on bowel care and in all cases made it easier, as in previous studies. 5 The one patient who felt that his quality of life was worse was troubled with constipation, although his time spent on bowel care had decreased from $10 \mathrm{~h}$ to $15 \mathrm{~min}$ and he did not want the stoma reversed. The only patient who was not 
independent in bowel care following stoma formation was a $\mathrm{C} 4$ tetraplegic.

The most common complication following colostomy formation was exclusion colitis. In one patient this was severe enough to warrant excision of the rectal stump which led to its own complications.

The results of our experience with colostomy in 12 spinal cord injured patients was very similar to that of Stone et al. ${ }^{6}$ We did not perform intestinal transit time or anorectal manometry although Stone et al felt that they were helpful in some patients. There is much debate as to whether these are required, or whether proceeding on clinical grounds, as in our study, is sufficient.

After spinal cord injury, most patients cope satisfactorily with bowel care for several years with a variety of regimes. This study has shown that the mean period of time from injury to stoma formation was 15 years. However the mean period of unsatisfactory bowel management before undergoing stoma formation was 5.4 years. This suggests that earlier intervention in patients with severe problems of bowel management may be beneficial.

By the end of the study period in 1996 there were almost 1200 patients registered with the Spinal Treatment Centre at Salisbury. As 14 intestinal stomas have been performed this corresponds to less than $1 \%$ of these patients. Although this is a relatively small figure the Spinal Centre only opened in 1984 and so the majority of patients have not had time to develop major problems of bowel care.

\section{Conclusion}

We conclude that colostomy is a safe, effective and well accepted treatment for selected patients with intract- able problems of bowel management following spinal cord injury. Ileostomy can also be effective but we believe that colostomy should be the preferred operation.

Our study suggests that colostomy should be more widely offered to spinal cord injury patients, not just those who have developed complications demanding stoma formation, but to patients with overwhelming and time-consuming problems of bowel care.

\section{References}

1 Glickman S, Kamm M. Bowel dysfunction in spinal cord injury patients. Lancet 1996; 347: $1651-1653$.

2 Menter R et al. Bowel management outcomes in individuals with long-term spinal cord injuries. Spinal Cord 1997; 35: 608-612.

3 Neilsen $\mathbf{J}$ et al. Constipation and incontinence in patients with spinal cord lesions. Presented at International Medical Society of Paraplegia meeting. Atlanta 1996.

4 Han TR, Kim JH, Kwon BS. Chronic gastrointestinal problems and bowel dysfunction in patients with spinal cord injury. Spinal Cord 1998; 36: $485-490$.

5 Craven ML, Etchells J. A review of the outcome of stoma surgery on spinal cord injured patients. Journal of Advanced Nursing 1998; 27: $922-926$.

6 Stone JM, Wolfe VA, Nino-Murcia M, Perkash I. Colostomy as treatment for complications of spinal cord injury. Arch Phys Med Rehabil 1990; 71: 514-518.

7 Macdough R et al. Control of defaecation in patients with spinal injury by stimulation of sacral anterior nerve roots. British Medical Journal 1990; 300: 1494 - 1497.

8 Hill J. Antegrade enemas for treatment of severe idiopathic constipation. British Journal of Surgery 1994; 81: 1490-1491. 